\title{
Unusual presentation of mucormycosis mimicking a localised sino-orbital pathology
}

\author{
Anuj Dadhich, ${ }^{1}$ Kumar Nilesh, ${ }^{2}$ Rahul Patil, ${ }^{3}$ Harish Saluja (1) ${ }^{4}$
}

'Department of Oral and Maxillofacial Surgery, Pravara Institute of Medical Sciences, Loni, Maharashtra, India ${ }^{2}$ Oral and Maxillofacial Surgery, Krishna Institute of Medical Sciences Deemed University, Karad, India

${ }^{3}$ Oral Pathology, Pravara Institute of Medical Sciences, Loni, Maharashtra, India ${ }^{4}$ OMFS, Pravara Rural Dental College, Ahmednagar, Maharashtra, India

Correspondence to Dr Kumar Nilesh; drkumarnilesh@yahoo.com

Accepted 22 December 2020

Check for updates

(C) BMJ Publishing Group Limited 2021. No commercial re-use. See rights and permissions. Published by BMJ.

To cite: Dadhich A, Nilesh $\mathrm{K}$, Patil $\mathrm{R}$, et al. BMJ Case Rep 2021;14:e239199. doi:10.1136/bcr-2020239199

\section{SUMMARY}

Mucormycosis is an aggressive and deadly fungal infection, which is invariably associated with an immunocompromised patient. Mucormycosis in the head and neck region presents as skeletal necrosis, with or without soft tissue involvement. Early identification and treatment with combination of surgical debridement and parenteral antifungal therapy is critical for a favourable outcome. This paper reports an unusual presentation of mucormycosis, mimicking a localised sino-orbital pathology involving the infraorbital subcutaneous tissue and the maxillary sinus, in a 35 years old immunocompetent man. Despite aggressive antifungal therapy and surgical management, the course of disease was fatal, reiterating the high mortality associated with mucormycosis.

\section{BACKGROUND}

Mucormycosis is a fungal infection which is relatively rare in in the maxillofacial region. Fungal species such as rhizopus, rhizomucor and mucor lead to development of mucormycosis. ${ }^{1}$ The fungi and its spores are present in the inhaled air and colonise the paranasal sinuses in otherwise healthy individuals. Under appropriate condition the fungi become saprophytic and rapidly multiply leading to an invasive infection, especially in immunocompromised host. ${ }^{23}$ The disease follows an aggressive course and extend rapidly into the neighbouring tissues leading to widespread necrosis of bone and soft tissue of oral cavity and face. Orbital involvement and cerebral invasion are common in advanced cases. These fungal infections demand prompt surgical intervention and prolonged antifungal therapy. Despite aggressive treatment the mortality rate remains as high as $80 \%{ }^{1}$

Localised form of the disease is rare and has almost exclusively been reported in immunocompetent individuals. ${ }^{4}$ This paper presents a case of mucormycosis, in an otherwise immunocompetent patient. The unusual presentation of the disease as a localised pathology, resulted in initial misdiagnosis. Definitive diagnosis of mucormycosis was made after the histopathological examination of the soft tissue lesion. Despite subsequent surgery, antifungal therapy and supportive medical care, the course of disease was fatal.

\section{CASE PRESENTATION}

A 35-year-old male patient was referred to maxillofacial surgery clinic with complaint of swelling in the left infraorbital region since past 3 months. Patient also gave a history of trauma over left midface region 4 years ago, for which no active intervention was done. Medical history of the patient was unremarkable. The patient was apparently all right 3 months ago when he noticed a peanut-sized painless swelling in the left infraorbital region, which gradually increased to the current size. Clinical examination revealed a firm, nodular swelling in the left infraorbital region which was approximately 2 $\mathrm{cm} \times 2 \mathrm{~cm}$ in size (figure $1 \mathrm{~A}$ ). The swelling was non-tender on palpation. Vision in the left eye was normal and there was no limitation in the movement of the eye ball. There was paraesthesia over the area of distribution of left infraorbital nerve. The overall clinical presentation was suggestive of a benign subcutaneous pathology. A provisional diagnosis of schwannoma involving the infraorbital nerve was made. The differential diagnosis included foreign body granuloma, lipoma, dermoid/epidermoid cyst and fibroma.

\section{INVESTIGATIONS}

MRI was performed for further evaluation of the lesion. MRI revealed a well-defined, intensity enhancing lesion involving the anterior wall of the left maxillary sinus. The lesion extends into the left orbital floor. The lesion was $3.2 \mathrm{~cm} \times 2.5 \mathrm{~cm} \times$ $2.3 \mathrm{~cm}$ in size and isointense on $\mathrm{T} 1$ and hypointense on $\mathrm{T} 2$ weighted images (figure 1B,C).

An incision biopsy of the lesion was planned and executed under local anaesthesia. The lesion was approached using an infraorbital incision and the specimen was submitted for histopathological evaluation. The tissue samples sent for diagnosis were examined grossly and processed for routine paraffin sections. These tissue preparations were stained with H\&E and Gomori's methenamine silver (GMS). H\&E stained sections showed aggregation of mononuclear round vacuolated cells, numerous multinucleated giant cells with eosinophillic cytoplasm, areas of granuloma formation

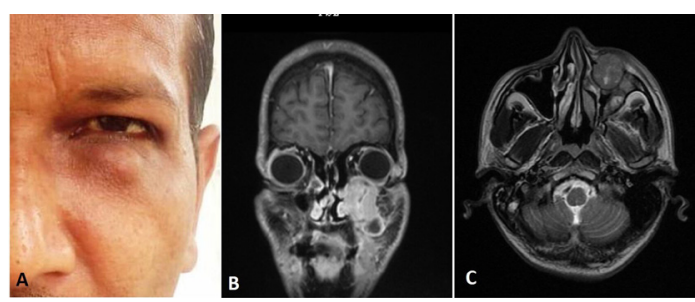

Figure 1 (A) Preoperative clinical photograph showing localised subcutaneous swelling in left infraorbital area and MRI (B) coronal and (C) axial sections showing well-defined lesion involving the anterior wall of the left maxillary sinus. 


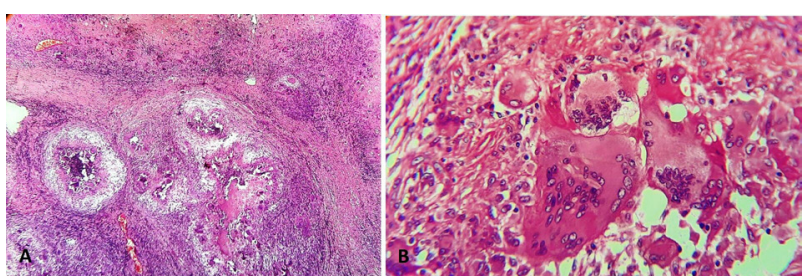

Figure 2 H\&E stained sections showing multiple caseating granulomas with numerous giant cells (10x) (A) and foreign body type of giant cells with fungal elements (40x) (B).

and intense chronic inflammatory cells chiefly lymphocytes and plasma cells. Giant cells had amorphous eosinophilic cytoplasm with multiple nuclei, distributed peripherally and eccentrically. Fungal elements were seen around the giant cells and near the blood vessels. Fungal elements demonstrate characteristic wide, ribbon-like, aseptate hyphae with right angle branching (figure 2). Special staining with GMS demonstrated fungal elements within the necrotic material and giant cells. Large nonsepta hyphae with right angle branching and non-parallel walls were identified. Angioinvasive causing tissue necrosis and haemorrhage was seen in the stained specimen (figure 3). The overall histological findings were suggestive of mucormycosis. Routine blood investigations, including complete haemogram and serum glucose level were within normal range. Laboratory examination showed no underlying immune deficiency.

\section{TREATMENT}

Following the diagnosis of mucormycosis, patient was hospitalised. Owing to greater risk of dissemination of the fungal infection from the infraorbital region and possible orbital and cerebral spread high dose $(0.7 \mathrm{mg} / \mathrm{kg} /$ day $)$ amphotericin B was started through intravenous infusion. Amphotericin B is polyene group of antifungal agents and is the most commonly used drug against mucormycosis. The starting dose of amphotericin B is $0.7 \mathrm{mg} / \mathrm{kg} /$ day. When required the dose can be titrated to 1 to $1.5 \mathrm{mg} / \mathrm{kg} /$ day, to a cumulative dose of 2 to $4 \mathrm{gm}$ over a period of weeks to months. The patient was kept under critical evaluation to monitor the response to antifungal therapy. Serum urea and creatinine levels were monitored and were found to be within normal range during the course of antifungal therapy.

Excision of the lesion was planned and executed under general anaesthesia. Weber Ferguson incision was used to gain access to the underlying lesion, which was the carefully excised in-toto, without causing any damage to the underlying vital structures (figure 4A, B and C). Closure was done in layers (figure 4D). The excised specimen was sent for histopathological evaluation. Histological findings were consistent with diagnosis of mucormycosis.

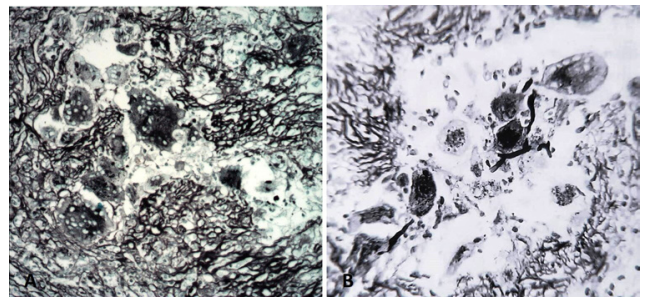

Figure 3 Gomori methamine silver stained photomicrograph demonstrating fungal elements within the necrotic material and giant cells, seen in 10x (A) and 40x magnification (B).

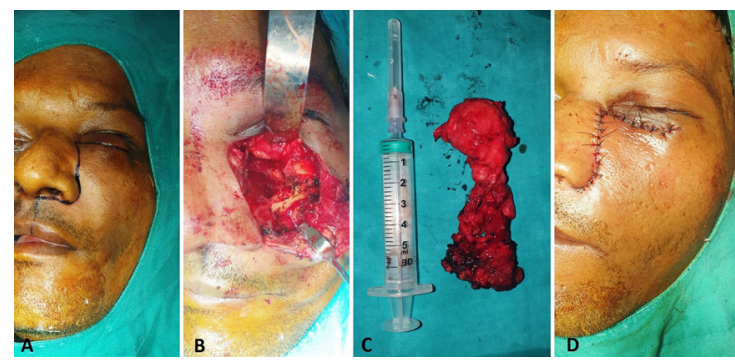

Figure 4 Intraoperative images showing $(A)$ incision marking, $(B)$ surgical site, (C) excised lesion and (D) closure.

\section{OUTCOME AND FOLLOW-UP}

Four days postoperatively the patient developed slurring of speech and giddiness. An immediate neuro-physician's opinion was taken and MRI was performed to rule out possible stroke or cerebral involvement due to mucormycosis. MRI revealed an acute non-haemorrhagic infarct in the left thalamo-capsular region suggestive of acute lacunar stroke. Patient was administered intravenous low molecular weight heparin (injection enoxaparin $1 \mathrm{mg} / \mathrm{kg} 12$ hourly) and vancomycin ( $20 \mathrm{mg} / \mathrm{kg} 6$ hourly). Cerebrospinal fluid (CSF) sample was taken for biochemical evaluation. After 3 days of intravenous low molecular weight heparin administration, patient showed significant improvement in speech and other neurological symptoms. CSF examination revealed increased values of proteins and glucose. Adinosine deaminase activity (ADA-MTB) activity was normal. Following improvement in clinical symptoms, low molecular weight heparin was stopped. Five days later the patient developed highgrade fever and stiffness in the neck. Cerebral dissemination of mucormycosis, resulting in meningitis was suspected. MRI was performed, which demonstrated multiple small areas of acute infarcts and intracerebral haemorrhage in left thalamus, intraventricular and subarachnoid haemorrhage. Amphotericin B therapy with dose of $0.7 \mathrm{mg} / \mathrm{kg} /$ day intravenous infusion was continued, with a total cumulative does of $0.6 \mathrm{gm}$. However, due to inadequate response to amphotericin $\mathrm{B}$ alone, salvage therapy was initiated with addition of azole group of antifungal agents. Various azole group of antifungal drugs have been reportedly used as second-line therapy with varying efficacy. While voriconazole and fluconazole do not show reliable activity against mucormycosis, activity of itraconazole is primarily reported against the Absidia species. Itraconazole was given in dose of $200 \mathrm{mg}$ every 12 hourly. However, on the second day the patient became comatose with no neurological response and 24 hours later succumbed to the disease.

\section{DISCUSSION}

Mucormycosis is primarily caused by mucorale Rhizopus oryzae. It is a non-septate filamentous fungus which is commonly found in soil, decaying fruits and vegetables, animal faeces. ${ }^{5}$ The most common means of acquiring infections in humans are through airborne fungal spores, ingestion and direct inoculation on traumatised tissue. ${ }^{6}$ In healthy individuals neutrophils play an important role in providing immunity against fungal infections. Conditions such as diabetes mellitus, cancer chemotherapy, presence of malignancy and immune compromised conditions can lead to severe neutropaenia, which can lead to rapid proliferation of the organism in human tissues. ${ }^{89}$ Other risk factors include; haemochromatosis, intravenous drug abuse, bone marrow transplantation, solid organ transplants, prolonged use of steroids or immunosuppressive therapy. ${ }^{10} 11$ While mucormycosis affects an 
individual, a predisposing medical condition is almost always present. Uncontrolled diabetes mellitus is the most common predisposing condition for development of mucormycosis. In these individuals granulocyte phagocytic activity is severely compromised and polymorphonuclear response is altered. ${ }^{9}$ Uncontrolled diabetes is associated with almost $40 \%$ of all head and neck mucormycosis cases and $70 \%$ of all rhino-orbitalcerebral mucormycosis. ${ }^{12}$ In the present case the patient was immunocompetent with no underlying risk factors like diabetes mellitus. Previous trauma over the affected site could have possibly been the possible portal of entry for the microorganism. However, this cannot be said with absolute surety.

Mucormycosis in head and neck region usually begins as a disease of nose or maxillary bone, and spread to the paranasal sinuses by direct extension. From paranasal sinuses the infection can directly spread to retro-orbital region or through lacrimal, angular and ethmoid vessels via haematogenous spread the fungus can disseminate in to brain and lungs. ${ }^{13}$ Colonisation of mucor hyphe in the blood vessels lead to formation of thrombi and reduce the vascularity of vital organs leading to development of necrosis. ${ }^{14}$ The most common clinical features of mucormycosis include; widespread necrosis of facial bones, pain and pus discharge from nose or eye. Orbital involvement can cause paresis of II, III, IV and VI cranial nerve, proptosis, ptosis, papillary dilation, visual impairment and blindness. ${ }^{15}$ Unlike the typical widespread and severe nature of presentation of mucormycosis, relatively localised and benign lesions have been sparingly reported. Nilesh reported a case of mucormycosis limited to right maxillary alveolus in an immunocompetent patient. ${ }^{16}$ In the present report, the lesion was typically localised as subcutaneous swelling in the infraorbital region, with paresthesia in the area of distribution of the infraorbital nerve. Such presentation can often lead to clinical misdiagnosis as it typically resembles a localised benign pathology, like schwannoma involving the terminal branches of the trigeminal nerve. ${ }^{17}$

CT and MRI play a vital role in diagnosis of mucormycosis in head and neck region. Radiographically it appears as nodular thickening of the sinus mucosa, sinus opacification with or without fluid accumulation, destruction of walls of paranasal sinuses and orbital floor. ${ }^{18}$ MRI provides better details of posterior orbit, optic canal, cavernous sinus and meningeal involvement. ${ }^{19}$ The confirmation of diagnosis of mucormycosis is made by histopathological evaluation of the specimen. Microscopic demonstration of aseptate hyphae with right-angled branching pattern is characteristic of mucormycosis. Mucorales can be cultured from specimen taken from involved skin or mucosal tissues. However, cultures are often negative and therefore are not always reliable to make the diagnosis. ${ }^{20}$ GMS stain has been used most effectively for the diagnosis. H\&E, periodic acidSchiff or calcofluor white stains may also be used. ${ }^{21}$

Once the diagnosis of mucormycosis is made, early and aggressive medical therapy with parenteral antifungal agent, surgical debridement of necrotic tissue and correction of underlying immunocompromised status are of utmost importance, so as to improve the outcome and minimise the morbidity. Despite aggressive and early management, mortality rate reported with mucormycosis is high, ranging from $60 \%$ to $80 \%$. $^{122}$

The ideal therapeutic approach for treatment of mucormycosis include; early diagnosis, treatment of underlying predisposing factors, aggressive debridement of necrotic tissue, establishment of adequate sinus drainage and aggressive and prolonged antifungal therapy. ${ }^{2324}$ There are several antifungal agents which can be used in treatment of mucormycosis. Pharmacologically they cause inhibition of synthesis or affect the integrity of ergosterol, the major sterol that forms fungal cell wall membranes. ${ }^{25}$ The most commonly used agents include; amphotericin B, fluconazole, ketoconazole, itraconazole, voriconazole, clotrimazole and posaconazole. ${ }^{24}$

Amphotericin B is the most commonly used drug against mucormycosis. The starting dose of amphotericin B is $0.7 \mathrm{mg} /$ $\mathrm{kg} /$ day, which can be increased to $1 \mathrm{mg} / \mathrm{kg} / \mathrm{day}$, to a cumulative dose of 2 to $4 \mathrm{gm}$ over a period of weeks to months. ${ }^{26}$ The most common adverse effects of amphotericin B include; fever, chills, headache, myalgia, anorexia, malaise, anaemia, hypokalemia and vomiting. Nephrotoxicity is a serious dose dependant side effect associated with use of amphotericin B, therefore blood urea, nitrogen and creatinine should be closely monitored. If blood urea nitrogen and creatinine exceeds beyond $50 \mathrm{mg} / \mathrm{dL}$ and $3.0 \mathrm{gm} / \mathrm{dL}$, respectively, then the dose should be administered on alternate days. ${ }^{27}$ If the levels continue to remain elevated, the dosage and frequency should be further reduced. Liposomal amphotericin B are newer agents with fewer side effects and are more potent in treatment of mucormycosis. They are the drug of choice in management of mucormycosis, have increased efficacy and decreased renal toxicity. ${ }^{26} 27$

Other antifungal agents used include posaconazole and itraconazole. Activity of itraconazole is primarily against the Absidia species of the fungi. ${ }^{27}$ Wide local excision and aggressive debridement is necessary to reduce the fungal burden in mucormycosis. The infected tissue bleeds less due to presence of ischaemia and therefore debridement should be done until the bleeding tissue is encountered. Repeated surgical intervention is indicated in many cases due to persistence of fungal load in the tissue. Orbital exenteration may be indicated when the orbit is inflamed with blindness or in cases of advanced orbital involvement. ${ }^{28}$

The fungal infection with mucormycosis in head and neck region are relatively rare. These infections are associated with wide spread necrosis of facial skeleton and associated soft tissue. It shows very high mortality rate, especially in immunocompromised individuals. Localised disease of mucormycosis has been inconsistently reported, and can often result in clinical misdiagnosis, leading to initial delay in appropriate treatment of this aggressive infection. ${ }^{13}{ }^{17}$ This case highlights initial localised presentation of rhino-orbital mucormycosis, which later had a fulminant course resulting in mortality. Physicians and surgeons

Learning points

- Mucormycosis is a rare fungal infections caused by rhizopus, rhizomucor and mucor. The fungi and its spores are present in the inhaled air. In an immunocompromised host it can become saprophytic and rapidly multiply leading to rapidly progressive necrosis of facial bones and soft tissues.

- Dissemination of infection to orbit, paranasal sinus and brain is common, if it is not treated early and aggressively.

- Mortality, as high as $60 \%$ to $80 \%$ is associated with mucormycosis of head and neck region.

- Localised presentation of mucormycosis has been infrequently reported and is commonly seen in individuals with no systemic comorbidities, like diabetes mellitus. Through this case report, the paper intends to highlight unusual presentation of mucormycosis, mimicking a localised pathology in infraorbital region. Such rare presentation can result in misdiagnosis and delay early treatment, which is critical for successful outcome. 
dealing with head and neck lesions should be aware of this rare disease and its unusual presentation, to initiate prompt treatment for favourable outcome.

Contributors $A D$ and $K N$ were involved in patient management and manuscript preparation. RP did the histology study assessment, while RP and HS were involved in final manuscript correction.

Funding The authors have not declared a specific grant for this research from any funding agency in the public, commercial or not-for-profit sectors.

Competing interests None declared.

Patient consent for publication Obtained.

Provenance and peer review Not commissioned; externally peer reviewed.

ORCID iD

Harish Saluja http://orcid.org/0000-0002-9511-083X

\section{REFERENCES}

1 Bhansali A, Bhadada S, Sharma A, et al. Presentation and outcome of rhino-orbitalcerebral mucormycosis in patients with diabetes. Postgrad Med J 2004;80:670-4.

2 Shamim MS, Siddiqui AA, Enam SA, et al. Craniocerebral aspergillosis in immunocompetent hosts: surgical perspective. Neurol India 2007;55:274-81.

3 Roden MM, Zaoutis TE, Buchanan WL, et al. Epidemiology and outcome of zygomycosis: a review of 929 reported cases. Clin Infect Dis 2005;41:634-53.

4 Nilesh $\mathrm{K}$, Vande AV. Mucormycosis of maxilla following tooth extraction in immunocompetent patients: reports and review. J Clin Exp Dent 2018;10:e300-5.

5 Kirszrot J, Rubin PAD. Invasive fungal infections of the orbit. Int Ophthalmol Clin 2007:47:117-32.

6 Sugar AM. Mucormycosis. Clin Infect Dis 1992;14:S126-9.

7 Saluja H, Rudagi BM, Sachdeva S, et al. Paranasal Aspergillus fungal infection in immune compromised and uncontrolled diabetic patients: a report of 5 cases along with review of literature. Clinics in surgery 2017:2:1-4.

8 Pak J, Tucci VT, Vincent AL, et al. Mucormycosis in immunochallenged patients. J Emerg Trauma Shock 2008;1:106-13.

9 Khandelwal P, Saluja H, Shah S, et al. Diabetic maxillary osteomyelitis: a worrisome Vulnerability—Our experience. J Maxillofac Oral Surg 2020;2 https://doi.org/

10 Gamaletsou MN, Sipsas NV, Roilides E, et al. Rhino-orbital-cerebral mucormycosis. Curr Infect Dis Rep 2012;14:423-34. -

11 Wali U, Balkhair A, Al-Mujaini A. Cerebro-rhino orbital mucormycosis: an update. J Infect Public Health 2012;5:116-26.
12 Auluck A. Maxillary necrosis by mucormycosis. A case report and literature review. Med Oral Patol Oral Cir Bucal 2007:12:E360-4.

13 Nilesh K, Malik NA, Belgaumi U. Mucormycosis in a healthy elderly patient presenting as oro-antral fistula: report of a rare incidence. J Clin Exp Dent 2015;7:e333-5.

14 Salisbury PL, Caloss R, Cruz JM, et al. Mucormycosis of the mandible after dental extractions in a patient with acute myelogenous leukemia. Oral Surg Oral Med Oral Pathol Oral Radiol Endod 1997:83:340-4.

15 Tugsel Z, Sezer B, Akalin T. Facial swelling and palatal ulceration in a diabetic patient. Oral Surg Oral Med Oral Pathol Oral Radiol Endod 2004;98:630-6.

16 Nilesh K, Vande AV. Mucormycosis of maxilla following tooth extraction in immunocompetent patients: reports and review. J Clin Exp Dent 2018;10:e300-5.

17 Kumar N. Infra-orbital nerve schwannoma: report and review. J Nat Sci Biol Med 2015;6:267-71.

18 Van der Westhuijzen AJ, Grotepass FW, Wyma G, et al. A rapidly fatal palatal ulcer: rhinocerebral mucormycosis. Oral Surg Oral Med Oral Pathol 1989;68:32-6.

19 Dyken ME, Biller J, Yuh WT, et al. Carotid-cavernous sinus thrombosis caused by Aspergillus fumigatus: magnetic resonance imaging with pathologic correlation--a case report. Angiology 1990;41:652-7.

20 Ferry AP, Abedi S. Diagnosis and management of rhino-orbitocerebral mucormycosis (phycomycosis). A report of 16 personally observed cases. Ophthalmology 1983;90:1096-104.

21 Tugsel Z, Sezer B, Akalin T. Facial swelling and palatal ulceration in a diabetic patient. Oral Surg Oral Med Oral Pathol Oral Radiol Endod 2004;98:630-6.

22 Altini C, Niccoli Asabella A, Ferrari C, et al. (18)F-FDG PET/CT contribution to diagnosis and treatment response of rhino-orbital-cerebral mucormycosis. Hell I Nucl Med 2015; 18:68-70

23 Yohai RA, Bullock JD, Aziz AA, et al. Survival factors in rhino-orbital-cerebral mucormycosis. Surv Ophthalmol 1994;39:3-22.

24 Chamilos G, Lewis RE, Kontoyiannis DP. Delaying amphotericin B-based frontline therapy significantly increases mortality among patients with hematologic malignancy who have zygomycosis. Clin Infect Dis 2008;47:503-9.

25 Pelton RW, Peterson EA, Patel BC, et al. Successful treatment of rhino-orbital mucormycosis without exenteration: the use of multiple treatment modalities. Ophthalmic Plast Reconstr Surg 2001;17:62-6.

26 Sun H-Y, Singh N. Mucormycosis: its contemporary face and management strategies. Lancet Infect Dis 2011:11:301-11.

27 Baginski M, Czub J. Amphotericin B and its new derivatives - mode of action. Curr Drug Metab 2009;10:459-69.

28 Nithyanandam S, Jacob MS, Battu RR, et al. Rhino-orbito-cerebral mucormycosis. A retrospective analysis of clinical features and treatment outcomes. Indian J Ophthalmol 2003;51:231-6.

Copyright 2021 BMJ Publishing Group. All rights reserved. For permission to reuse any of this content visit

https://www.bmj.com/company/products-services/rights-and-licensing/permissions/

BMJ Case Report Fellows may re-use this article for personal use and teaching without any further permission.

Become a Fellow of BMJ Case Reports today and you can:

- Submit as many cases as you like

- Enjoy fast sympathetic peer review and rapid publication of accepted articles

- Access all the published articles

Re-use any of the published material for personal use and teaching without further permission

Customer Service

If you have any further queries about your subscription, please contact our customer services team on +44 (0) 2071111105 or via email at support@bmj.com.

Visit casereports.bmj.com for more articles like this and to become a Fellow 